\title{
Two-Loop Massive Operator Matrix Elements for Polarized and Unpolarized Deep-Inelastic Scattering
}

\author{
I. Bierenbaum;, J. Blümlein and S. Klein \\ Deutsches Elektronen-Synchrotron, DESY, Platanenallee 6, D-15738 Zeuthen, Germany \\ E-mail: Isabella.Bierenbaum@desy.de, Johannes. Blümlein@desy.de, \\ Sebastian.Klein@desy.de
}

\begin{abstract}
We calculate the $O\left(\alpha_{s}^{2}\right)$ gluonic massive operator matrix elements for unpolarized and polarized heavy flavor production, at asymptotic values $Q^{2} \gg m^{2}$. This calculation is done in Mellin space and without applying the integration-by-parts method, which was used in a former $x$-space calculation in Ref. [1], the results of which we confirm. However, our results present themselves in a more compact way, needing a basis of only 2 compared to 48 basic functions in the unpolarized case, and 24 in the polarized case. The results are obtained in two ways, by hypergeometric functions and by the use of Mellin-Barnes integrals, on which we put the emphasis in this paper.
\end{abstract}

XI International Workshop on Advanced Computing and Analysis Techniques in Physics Research April 23-27 2007

Amsterdam, the Netherlands

${ }^{*}$ Speaker 


\section{Introduction}

The contributions of the heavy-flavor corrections to the unpolarized deep-inelastic structure functions in the range of small values of Bjorken- $x$ are on the level of 20-40\%. Hence, the knowledge of these contributions to high accuracy is of importance for precision measurements of $\Lambda_{\mathrm{QCD}}$ [2] and the parton distributions. For the general kinematic range, the next-to-leading order corrections were given semi-analytically in [3], with a fast implementation of these corrections in Mellin-space given in [4].

In the region $Q^{2} \gg m^{2}, Q^{2}$ denoting the virtuality of the gauge boson exchanged in deep-inelastic scattering, and $m$ the mass of the heavy quark under consideration, the heavy flavor Wilson coefficients were derived analytically to $O\left(\alpha_{s}^{2}\right)$ in [1,5]. These calculations were done in $x$-space using the integration-by-parts method, making the integrals obtained more easy to solve, however, leading to a proliferation of a huge set of terms. Here, we summarize the results of a first re-calculation of the unpolarized and polarized $O\left(\alpha_{s}^{2}\right)$ gluonic massive operator matrix elements (OMEs) carried out in $[6,7]$. The unpolarized and polarized massive operator matrix elements can be used to calculate the asymptotic heavy-flavor Wilson coefficients for $F_{2}\left(x, Q^{2}\right)$ and $g_{1}\left(x, Q^{2}\right)$ to $O\left(\alpha_{s}^{2}\right)[1,5-7]$, and for $F_{L}\left(x, Q^{2}\right)$ to $O\left(\alpha_{s}^{3}\right)$ [8]. In contrast to the work in [1,5], our calculation is performed in Mellin space using harmonic sums $[9,10]$, without applying the integration-by-parts technique. In this way, we can significantly compactify both, the intermediary and final results, which are found to be in agreement with the results obtained in $[1,5]$. The calculation was done using two methods: On the one hand we used generalized hypergeometric functions to express and expand the integrals in $\varepsilon=D-4$, on the other hand we used the Mellin-Barnes technique (see e.g. [11,12]).

In this paper, we particularly emphasize the use of Mellin-Barnes integrals and the fact that the calculation was done in Mellin space. The method of Mellin-Barnes integrals was in our calculations mainly used to obtain numeric results rather than analytic results. However, the appearance of Mellin-Barnes integrals in many calculations throughout the last years [13] and the fact that they provide a numeric check on our results, motivates having a closer look at this method.

\section{Mellin space and Mellin-Barnes integrals}

The Mellin-transform $\mathbf{M}[f, s]$ of a function $f$ is given by the integral (cf. e.g. [14], and References therein):

$$
F(s)=\mathbf{M}[f, s]=\int_{0}^{\infty} x^{s-1} f(x) d x, \quad a<\operatorname{Re}(s)<b,
$$

under the condition that $f(x)$ falls down rapidly enough at the boundaries. The mathematical inverse of this function is given by the following integral, which is also called a Mellin-Barnes integral:

$$
f(x)=\frac{1}{2 \pi i} \int_{c-i \infty}^{c+i \infty} x^{-s} F(s) d s, \quad a<c<b .
$$

An important example of a Mellin-transform and its inverse, which we will make use of in the following, is given by the Euler-Beta function (cf. [14]):

$$
\mathbf{M}[f, s]=\frac{\Gamma(s) \Gamma(t)}{\Gamma(s+t)}=\int_{0}^{\infty} \frac{x^{s-1}}{(1+x)^{s+t}} d x, \quad(\operatorname{Re}(s, t)>0) .
$$


From this we deduce that

$$
f(x)=\frac{1}{(1+x)^{v}}=\frac{1}{2 \pi i} \int_{c-i \infty}^{c+i \infty} x^{-s} \frac{\Gamma(s) \Gamma(v-s)}{\Gamma(v)} d s, \quad 0<c<\operatorname{Re}(v) .
$$

The integration contour is placed at a value $c$, such that it separates the poles of the $\Gamma$-function $\Gamma(s)$ from those of the function $\Gamma(v-s)$. A generalization of this formula is used in the calculation of the two-loop massive OMEs in the next section.

In our context, we encounter the Mellin transformation in deep-inelastic scattering, where the integral is given over the Bjorken variable, $x=Q^{2} / 2 p q$, which runs between $0 \leq x \leq 1$. Hence, the Mellin-transform is given by the corresponding integral:

$$
F(N)=\mathbf{M}[f, N]=\int_{0}^{1} x^{N-1} f(x) d x .
$$

The convolution of two functions $f$ and $g$ is defined as:

$$
[f \otimes g](x)=\int_{0}^{1} d x_{1} \int_{0}^{1} d x_{2} \delta\left(x-x_{1} x_{2}\right) f\left(x_{1}\right) g\left(x_{2}\right) .
$$

One of the advantages of Mellin-space calculations is the fact that the Mellin-transform of a convolution of functions (see below) reduces to a simple product:

$$
\mathbf{M}[f \otimes g, N]=\mathbf{M}[f, N] \mathbf{M}[g, N]=F(N) G(N) .
$$

The Mellin-parameter $N$ appears in our results in form of harmonic sums of limit $N$. The general form of a harmonic sum is given by $[9,10]$ :

$$
\begin{aligned}
S_{a_{1}, \ldots, a_{m}}(N) & =\sum_{n_{1}=1}^{N} \sum_{n_{2}=1}^{n_{1}} \ldots \sum_{n_{m}=1}^{n_{m-1}} \frac{\left(\operatorname{sign}\left(a_{1}\right)\right)^{n_{1}}}{n_{1}^{\left|a_{1}\right|}} \frac{\left(\operatorname{sign}\left(a_{2}\right)\right)^{n_{2}}}{n_{2}^{\left|a_{2}\right|}} \ldots \frac{\left(\operatorname{sign}\left(a_{m}\right)\right)^{n_{m}}}{n_{m}^{\left|a_{m}\right|}}, \\
N & \in \mathbb{N}, \forall \ell, a_{\ell} \in \mathbb{Z} \backslash\{0\} .
\end{aligned}
$$

\section{The Method}

In the limit $Q^{2} \gg m^{2}$, one finds on the one hand the heavy flavor structure functions to be a convolution of the perturbatively calculable heavy flavor Wilson coefficients and the non-perturbative parton distribution functions. On the other hand, the heavy quark contributions to the twist-2 Wilson coefficients are determined by universal massive operator matrix elements $\left\langle i\left|A_{l}\right| j\right\rangle$ between partonic states. The process dependence enters through the corresponding massless Wilson coefficients [15]. This separation is obtained applying the renormalization group equation(s) to the (differential) scattering cross sections, cf. [1]. In this way all logarithmic and the constant contribution in $m^{2} / Q^{2}$ can be determined. The operator matrix elements are calculated applying the operator insertions due to the light-cone expansion in the respective amplitudes. One obtains the following convolution for the heavy flavor Wilson coefficients:

$$
H_{(2, L), i}^{\mathrm{S}, \mathrm{NS}}\left(\frac{Q^{2}}{\mu^{2}}, \frac{m^{2}}{\mu^{2}}\right)=\underbrace{A_{k, i}^{\mathrm{S}, \mathrm{NS}}\left(\frac{m^{2}}{\mu^{2}}\right)}_{\text {massive OMEs }} \otimes \underbrace{C_{(2, L), k}^{\mathrm{S}, \mathrm{NS}}\left(\frac{Q^{2}}{\mu^{2}}\right)}_{\text {light Wilson coefficients }} .
$$


Both convolutions cited above reduce to a pure product in Mellin space, as explained in the previous section. The OMEs contain ultraviolet and collinear divergences. The collinear singularities are absorbed into the parton distribution functions while the ultraviolet divergences are removed through renormalization. The $O\left(\alpha_{s}^{2}\right)$ coefficient of the perturbative expansion of the gluonic OME reads :

$$
\begin{aligned}
A_{Q g}^{(2)}= & \frac{1}{8}\left\{\widehat{P}_{q g}^{(0)} \otimes\left[P_{q q}^{(0)}-P_{g g}^{(0)}+2 \beta_{0}\right]\right\} \ln ^{2}\left(\frac{m^{2}}{\mu^{2}}\right)-\frac{1}{2} \widehat{P}_{q g}^{(1)} \ln \left(\frac{m^{2}}{\mu^{2}}\right) \\
& +\bar{a}_{Q g}^{(1)} \otimes\left[P_{q q}^{(0)}-P_{g g}^{(0)}+2 \beta_{0}\right]+a_{Q g}^{(2)},
\end{aligned}
$$

and similar for the quarkonic contributions. Here, $\mu^{2}$ denotes the factorization and renormalization scale, which are set to be equal, $P_{i j}^{(k-1)}$ are the $k$ th-loop splitting functions and $\beta_{0}$ denotes the lowest expansion coefficient of the $\beta$-function. $a_{i j}^{(k)}$ and $-q_{j}^{(k)}$ are the $O\left(\varepsilon^{0}\right), O(\varepsilon)$-terms resp., in the expansion of the OME, which form the main objective of the present calculation.

\section{Calculation}

Our calculation was done in two ways: On the one hand, after momentum integration, we expressed the Feynman-parameter integrals as hypergeometric functions and generalizations thereof, expanding these functions in $\varepsilon$ and solving for each desired order in $\varepsilon$ the corresponding sums. The results were further simplified using algebraic relations between harmonic sums [16]. On the other hand, we used Mellin-Barnes integrals as explained in [12a] for scalar integrals. Consider, for example, diagram I of [12a] and its corresponding integral after Wick rotation to Euclidean space:

$$
I_{e}:=\iint \frac{d^{D} q d^{D} k}{(2 \pi)^{2 D}} \frac{(\Delta \cdot q)^{N}}{\left[q^{2}+m^{2}\right]\left[(q-p)^{2}+m^{2}\right]\left[k^{2}+m^{2}\right]\left[(k-p)^{2}+m^{2}\right]\left[(k-q)^{2}\right]}
$$

The idea is following the gluing operation of graphs, as explained in [12a]. The result of the twopoint function with the three-point sub-diagram shrunk to a point is, using that $\Delta$ is a light-like vector with $\Delta^{2}=0$, easily evaluated to a simple fraction of $\Gamma$-functions:

$$
\int \frac{d^{D} q}{i \pi^{\frac{D}{2}}} \frac{\left(m^{2}\right)^{v_{14}-D / 2}(\Delta \cdot q)^{N}}{\left[q^{2}+m^{2}\right]^{v_{1}}\left[(q-p)^{2}+m^{2}\right]^{v_{4}}}=(\Delta \cdot p)^{N} \frac{\Gamma\left(v_{14}-D / 2\right) \Gamma\left(v_{4}+N\right)}{\Gamma\left(v_{4}\right) \Gamma\left(v_{14}+N\right)} .
$$

In a second step, one seeks for a way to rewrite and solve the integral for the three-point one-loop sub-diagram such that it can be combined with the two-point result. More precisely, one tries to express the following integral of the sub-diagram into the momentum invariants of (4.1):

$$
\begin{aligned}
& \int \frac{d^{D} k}{i \pi^{\frac{D}{2}}} \frac{\left[m^{2}\right]^{v_{235}-D / 2}}{\left[k^{2}+m^{2}\right]^{\nu_{2}}\left[(k-p)^{2}+m^{2}\right]^{\nu_{3}}\left[(k-q)^{2}\right]^{v_{5}}} \\
& \stackrel{!}{=} \frac{c(\Gamma)}{(2 \pi i)^{2}} \int_{\gamma_{1}-i \infty}^{\gamma_{1}+i \infty} d \sigma \int_{\gamma_{2}-i \infty}^{\gamma_{2}+i \infty} d \tau\left[\frac{(q-p)^{2}+m^{2}}{m^{2}}\right]^{\sigma}\left[\frac{q^{2}+m^{2}}{m^{2}}\right]^{\tau} \Gamma\left(\varepsilon, v_{i}, \sigma, \tau\right),
\end{aligned}
$$


where $\Gamma\left(\varepsilon, v_{i}, \sigma, \tau\right)$ is any fraction of $\Gamma$-functions occurring. This is achieved by first introducing Feynman parameters and in such expressing the product of propagators into a sum of terms:

$$
\frac{1}{A_{1}^{v_{1}} A_{2}^{v_{2}} \ldots A_{n}^{v_{n}}}=\int_{0}^{1} d x_{1} \ldots d x_{n} \delta\left(\sum x_{i}-1\right) \frac{\Pi x_{i}^{v_{i}-1}}{\left[x_{1} A_{1}+x_{2} A_{2}+\ldots+x_{n} A_{n}\right]^{\left[v_{i}\right.}} \frac{\Gamma\left(v_{1}+\ldots+v_{n}\right)}{\Gamma\left(v_{1}\right) \ldots \Gamma\left(v_{n}\right)}
$$

After doing the momentum integration as usual, one groups the expression in such a way that the $A_{i}$ are in the form of the momenta of the integral in (4.1). To this sum one then applies the Mellin-Barnes transformation, in this transforming the sum back into a product:

$$
\begin{aligned}
\frac{1}{\left(A_{1}+A_{2}+\ldots+A_{n}\right)^{v}} & =\frac{1}{(2 \pi i)^{n-1} \Gamma(v)} \int_{\gamma-i \infty}^{\gamma+i \infty} d \sigma_{1} \ldots \int_{\gamma-i \infty}^{\gamma+i \infty} d \sigma_{n-1} \\
& \times A_{1}^{\sigma_{1}} A_{2}^{\sigma_{2}} \ldots A_{n}^{-\sigma_{1} \ldots-\sigma_{n-1}-v} \Gamma\left(-\sigma_{1}\right) \ldots \Gamma\left(-\sigma_{n-1}\right) \Gamma\left(\sigma_{1}+\ldots+\sigma_{n-1}+v\right)
\end{aligned}
$$

In this sense, one can consider the Mellin-Barnes integral as the inverse of Feynman parametrization (cf. [17]).

With the Mellin-Barnes integrals obtained in this way, we then used the mathematica package MB [18], written by M. Czakon, to derive numeric results. In order to obtain analytic results, one can close the contour of the Mellin-Barnes integrals at infinity and use the residue theorem to express the integrals as sums over $\Gamma$-functions (cf. [11, 12]). However, for the general scalar integrals it turned out to be very difficult to solve the sums obtained by the method of residues, and therefore only a subset of all diagrams was calculated analytically in this way. Additionally, one does not only have genuine scalar two-loop integrals, but due to the numerator structure, one also encounters integrals where one or more of the propagators cancel (so-called reduced integrals), or appear in the numerator of the integral. Especially the reduced integrals were not always easily expressible as a Mellin-Barnes integral in our kinematics, however, could rather be solved by simply rewriting the corresponding Feynman parameter integral into Beta-functions.

\section{Results}

We calculated the gluonic massive operator matrix elements both, for the gluon-heavy quark and light-heavy quark transitions in the flavor non-singlet and singlet cases, for unpolarized and polarized nucleon targets. The constant contribution to the unpolarized and polarized OMEs for the transition $g \rightarrow Q$ are (cf. [6,7]): 


$$
\begin{aligned}
& a_{Q g}^{(2, \text { unpol })}(N)=4 C_{F} T_{R}\left\{\frac { N ^ { 2 } + N + 2 } { N ( N + 1 ) ( N + 2 ) } \left[-\frac{1}{3} S_{1}^{3}(N-1)+\frac{4}{3} S_{3}(N-1)-S_{1}(N-1) S_{2}(N-1)\right.\right. \\
& \left.-2 \zeta_{2} S_{1}(N-1)\right]+\frac{N^{4}+16 N^{3}+15 N^{2}-8 N-4}{N^{2}(N+1)^{2}(N+2)} S_{2}(N-1)+\frac{3 N^{4}+2 N^{3}+3 N^{2}-4 N-4}{2 N^{2}(N+1)^{2}(N+2)} \zeta_{2} \\
& \left.+\frac{2}{N(N+1)} S_{1}^{2}(N-1)+\frac{N^{4}-N^{3}-16 N^{2}+2 N+4}{N^{2}(N+1)^{2}(N+2)} S_{1}(N-1)+\frac{P_{1}(N)}{2 N^{4}(N+1)^{4}(N+2)}\right\} \\
& +4 C_{A} T_{R}\left\{\frac { N ^ { 2 } + N + 2 } { N ( N + 1 ) ( N + 2 ) } \left[4 \mathrm{M}\left[\frac{\mathrm{Li}_{2}(x)}{1+x}\right](N+1)+\frac{1}{3} S_{1}^{3}(N)+3 S_{2}(N) S_{1}(N)\right.\right. \\
& \left.+\frac{8}{3} S_{3}(N)+\beta^{\prime \prime}(N+1)-4 \beta^{\prime}(N+1) S_{1}(N)-4 \beta(N+1) \zeta_{2}+\zeta_{3}\right]-\frac{N^{3}+8 N^{2}+11 N+2}{N(N+1)^{2}(N+2)^{2}} S_{1}^{2}(N) \\
& -2 \frac{N^{4}-2 N^{3}+5 N^{2}+2 N+2}{(N-1) N^{2}(N+1)^{2}(N+2)} \zeta_{2}-\frac{7 N^{5}+21 N^{4}+13 N^{3}+21 N^{2}+18 N+16}{(N-1) N^{2}(N+1)^{2}(N+2)^{2}} S_{2}(N) \\
& -\frac{N^{6}+8 N^{5}+23 N^{4}+54 N^{3}+94 N^{2}+72 N+8}{N(N+1)^{3}(N+2)^{3}} S_{1}(N)-4 \frac{N^{2}-N-4}{(N+1)^{2}(N+2)^{2}} \beta^{\prime}(N+1) \\
& \left.+\frac{P_{2}(N)}{(N-1) N^{4}(N+1)^{4}(N+2)^{4}}\right\} \text {. } \\
& a_{Q g}^{(2, \mathrm{pol})}(N)=C_{F} T_{R}\left\{4 \frac{N-1}{3 N(N+1)}\left(-4 S_{3}(N)+S_{1}^{3}(N)+3 S_{1}(N) S_{2}(N)+6 S_{1}(N) \zeta_{2}\right)\right. \\
& -4 \frac{N^{4}+17 N^{3}+43 N^{2}+33 N+2}{N^{2}(N+1)^{2}(N+2)} S_{2}(N)-4 \frac{3 N^{2}+3 N-2}{N^{2}(N+1)(N+2)} S_{1}^{2}(N) \\
& \left.-2 \frac{(N-1)\left(3 N^{2}+3 N+2\right)}{N^{2}(N+1)^{2}} \zeta_{2}-4 \frac{N^{3}-2 N^{2}-22 N-36}{N^{2}(N+1)(N+2)} S_{1}(N)-\frac{2 P_{3}(N)}{N^{4}(N+1)^{4}(N+2)}\right\} \\
& +C_{A} T_{R}\left\{4 \frac { N - 1 } { 3 N ( N + 1 ) } \left(12 \mathrm{M}\left[\frac{\mathrm{Li}_{2}(x)}{1+x}\right](N+1)+3 \beta^{\prime \prime}(N+1)-8 S_{3}(N)-S_{1}^{3}(N)\right.\right. \\
& \left.-9 S_{1}(N) S_{2}(N)-12 S_{1}(N) \beta^{\prime}(N+1)-12 \beta(N+1) \zeta_{2}-3 \zeta_{3}\right)-16 \frac{N-1}{N(N+1)^{2}} \beta^{\prime}(N+1) \\
& +4 \frac{N^{2}+4 N+5}{N(N+1)^{2}(N+2)} S_{1}^{2}(N)+4 \frac{7 N^{3}+24 N^{2}+15 N-16}{N^{2}(N+1)^{2}(N+2)} S_{2}(N)+8 \frac{(N-1)(N+2)}{N^{2}(N+1)^{2}} \zeta_{2} \\
& \left.+4 \frac{N^{4}+4 N^{3}-N^{2}-10 N+2}{N(N+1)^{3}(N+2)} S_{1}(N)-\frac{4 P_{4}(N)}{N^{4}(N+1)^{4}(N+2)}\right\} \text {. }
\end{aligned}
$$

Here $P_{i}(N)$ denote polynomials given in [6,7]. The corresponding quarkonic expressions are given there.

Structural relations for harmonic sums [20], which include half-integer relations and differentiation w.r.t. the Mellin variable $N$, lead to the observation that the OMEs above depend only on the two basic harmonic sums $S_{1}(N)$ and $S_{-2,1}(N)$. We expressed the latter in terms of the Mellin transform $M\left[\operatorname{Li}_{2}(x) /(1+x)\right](N)$ in the above. Here $\beta(N)=(1 / 2) \cdot[\psi((N+1) / 2)-\psi(N / 2)]$. Previous analyzes of various other space- and time-like two-loop Wilson coefficients and anomalous dimensions including also the soft and virtual corrections to Bhabha-scattering [20a,21], showed that the following six basic functions, $S_{1}(N), S_{ \pm 2,1}(N), S_{-3,1}(N), S_{ \pm 2,1,1}(N)$, are needed in general to express these quantities. Note that none of the harmonic sums occurring contains an index $\{-1\}$ as observed in all other cases being analyzed. 
Comparing to the results obtained in Refs. [1,5] in $x$-space, we find 48 functions to express the final result in the unpolarized case and 24 functions in the polarized case.

To obtain expressions for the heavy-flavor contributions to the structure functions in $x$-space, analytic continuations have to be performed to $N \in \mathbf{C}$ for the basic functions given above, see [20, $22,23]$. Finally a (numeric) contour integral has to be performed around the singularities present.

\section{Conclusions}

We calculated the gluonic unpolarized and polarized massive operator matrix elements to $O\left(\alpha_{s}^{2}\right)$. They are needed to express the heavy flavor Wilson coefficients contributing to the deepinelastic structure functions $F_{2}, g_{1}$ and $F_{L}$ to $O\left(\alpha_{s}^{2}\right), O\left(\alpha_{s}^{3}\right)$ resp., in the region $Q^{2} \gg m^{2}$. The calculation was performed in Mellin space without using the integration-by-parts technique. The results express themselves in form of nested harmonic sums. Throughout the calculation, we applied representations by Mellin-Barnes integrals and generalized hypergeometric functions. In this paper, we emphasized the use of Mellin-Barnes integrals, which can be regarded as an inverse transformation of Feynman parametrization.

In course of the calculations, a series of new infinite sums over products of harmonic sums weighted by related functions were evaluated, cf. [6,7]. These representations were essential to keep the complexity of the intermediary and final results as low as possible. Furthermore, we applied a series of mathematic relations for the harmonic sums to compactify the results further. We confirm the results obtained earlier in Refs. [1,5] by other technologies.

\section{Acknowledgement}

This paper was supported in part by SFB-TR-9: Computergestütze Theoretische Teilchenphysik, and the Studienstiftung des Deutschen Volkes.

\section{Literatur}

[1] M. Buza, Y. Matiounine, J. Smith, R. Migneron and W. L. van Neerven, Nucl. Phys. B472 (1996) 611 [hep-ph/9601302].

[2] J. Blümlein, DESY 07-083, arXiv:0706.2430 [hep-ph];

J. Blümlein, H. Böttcher and A. Guffanti, Nucl. Phys. B774 (2007) 182 [hep-ph/ 0607200 ].

[3] E. Laenen, S. Riemersma, J. Smith and W. L. van Neerven, Nucl. Phys. B392 (1993) 162;

S. Riemersma, J. Smith and W. L. van Neerven, Phys. Lett. B347 (1995) 143 [hep-ph/ 9411431$].$

[4] S. I. Alekhin and J. Blümlein, Phys. Lett. B594 (2004) 299 [hep-ph / 0404034 ].

[5] M. Buza, Y. Matiounine, J. Smith and W. L. van Neerven, Nucl. Phys. B485 (1997) 420 [hep-ph/9608342].

[6] I. Bierenbaum, J. Blümlein and S. Klein, Nucl. Phys. B (2007) in print hep-ph/ 0703285.

[7] J. Blümlein and S. Klein, DESY 07-027.

[8] J. Blümlein, A. De Freitas, W. L. van Neerven and S. Klein, Nucl. Phys. B755 (2006) 272

[hep-ph/0608024]. 
[9] J. Blümlein and S. Kurth, Phys. Rev. D60 (1999) 014018 [hep-ph/9810241].

[10] J. A. M. Vermaseren, Int. J. Mod. Phys. A14 (1999) 2037 [hep-ph / 9806280$].$

[11] I. Bierenbaum and S. Weinzierl, Eur. Phys. J. C32 (2003) 67 [hep-ph/ 0308311$].$

[12] I. Bierenbaum, J. Blümlein and S. Klein, Nucl. Phys. Proc. Suppl. 160 (2006) 85 [hep-ph/0607300];

I. Bierenbaum, J. Blümlein and S. Klein, Phys. Lett. B648 (2007) 195 [hep-ph / 0702265$].$

[13] V. A. Smirnov, Phys. Lett. B460 (1999) 397 [hep-ph/9905323];

J. B. Tausk, Phys. Lett. B469 (1999) 225 [hep-ph/9909506];

V. A. Smirnov and O. L. Veretin, Nucl. Phys. B566 (2000) 469 [hep-ph / 9907385 ];

V. A. Smirnov, Phys. Lett. B547 (2002) 239 [hep-ph/ 0209193 ];

V. A. Smirnov, Nucl. Phys. Proc. Suppl. 135 (2004) 252 [hep-ph/ 0406052 ];

M. Czakon, J. Gluza and T. Riemann, Nucl. Phys. $B 751$ (2006) 1 [hep-ph/0604101].

[14] R.B. Paris, D. Kaminsky, Asymptotics and Mellin-Barnes Integrals (Encyclopedia of Mathematics and its Applications 85), Cambridge University Press, Cambridge 2001

[15] E. B. Zijlstra and W. L. van Neerven, Nucl. Phys. B383 (1992) 525;

E. B. Zijlstra and W. L. van Neerven, Nucl. Phys. B417 (1994) 61 [Erratum-ibid. B426 (1994) 245, 773 (2007) 105];

S. Moch, J. A. M. Vermaseren and A. Vogt, Phys. Lett. B606 (2005) 123 [hep-ph/0 411112 ].

[16] J. Blümlein, Comput. Phys. Commun. 159 (2004) 19 [hep-ph/0311046].

[17] S. Weinzierl, hep-ph/0604068.

[18] M. Czakon, Comput. Phys. Commun. 175 (2006) 559 [hep-ph/ 0511200 ].

[19] I. Bierenbaum, J. Blümlein and S. Klein, arXiv:0706.2738 [hep-ph] .

[20] J. Blümlein and S. Klein, DESY 07-082, arXiv:0706.2426 [hep-ph]; J. Blümlein, DESY 07-042.

[21] J. Blümlein and V. Ravindran, Nucl. Phys. B716 (2005) 128 [hep-ph/ 0501178 ];

J. Blümlein and V. Ravindran, Nucl. Phys. B749 (2006) 1 [hep-ph/ 0604019 ];

J. Blümlein and S. Moch, in preparation.

[22] J. Blümlein, Comput. Phys. Commun. 133 (2000) 76 [hep-ph/ 0003100 ].

J. Blümlein and S. O. Moch, Phys. Lett. B614 (2005) 53 [hep-ph/ 0503188$].$

[23] A. V. Kotikov and V. N. Velizhanin, hep-ph/0501274. 